\title{
Research on 3D Modeling Method Based on Hybrid Octree Structure
}

\author{
Wang Yujian*, Tan Shaowei, Dong Weiwei and Jing Wenpeng \\ College of Information Technology, Beijing Union University, Beijing 100101, China
}

\begin{abstract}
With studying deeply of the three-dimensional modeling method, this paper proposed a hybrid data model which based on Octree,the four fork tree and NURBS. The characteristic of fast convergence of Octree is used to segment the 3D entity. Describe the irregular surface of entity by NURBS, and restructure the local mesh surface. The model uses the mixture data structure of Octree and four fork tree to restructure mesh surface gradually. The storage structure is the Octree structure type; establish Hash table based on octal prefix code. Finally, an experimental model system is designed by using OpenGL. The feasibility and effectiveness of the algorithm has been verified.
\end{abstract}

Keywords: 3D modeling, NURBS, Octree, surface reconstruction.

\section{INTRODUCTION}

In recent years, people have been studying deeply of the three-dimensional modeling method; put forward a large number of methods of 3D solid modeling [1,2]. The 3D spatial data model is divided into body element model and surface model. The body element model is applied to Space operation and analysis, but the data structure is too complex and has a large storage space. Octree is a typical body element model. From the principle of construction of spatial data structures, Octree is very suitable for the classification of spatial data processing. Document [3] proposed a block model which based on Octree use the intersection detection unit and a surface model to the bounding box, convert complex orebody surface model and block model. This method can accurately establish block model of complex orebody from various surface model. Document [4] using spatially distributed method, this model distribute the massive data into multiple servers, and enhance the data management efficiency by using parallel access technology.

The storage capacity of surface-units model data is small, and this data has fast modeling speed, but it can't describe the internal property of entity, and it's hard to achieve the 3D spatial analysis and query. Surface reconstruction technology is the basic of element surface model, and it's a form to find some mathematical description, the mathematical model which is constructed corresponding to describe the real shape of the surface precisely and sententiously. Surface reconstruction is widely used in computer graphics, computer aided design, computer vision, reverse engineering and other fields [5]. In recent years, researchers have proposed many surface reconstruction algorithm which based on Delaunay, parametric or implicit surfaces [6]. These algorithms have characteristics of simple geometrical calculation, convenient display, no deformation, easy to control etc.. However, the most of these algorithms have many problems related to mesh and to uniform, which have increase the calculation of surface reconstruction. The effect of model reconstruction is not ideal on the structure of complex entity.

\section{OCTREE MODELING}

\subsection{Octree Structure}

Octree is a recursive data structures which is extended from the four fork tree to 3D space. As shown in Fig. (1), the Octree divided the 3D entity into 8 equal sized sub-cube according to $\mathrm{X}, \mathrm{Y}, \mathrm{Z}$ three directions. Then according to the target of each sub-cube to determine whether to continue to dividing the cube into 8 equal sized sub-cube, until each subcube has no goal, or full of target, or its size has become a pre-defined indivisible voxel. Each node of Octree has 8 sub node or no child nodes. Resolution of each dimension will be increased to two times by divide.

The Octree is divided into regular Octree and linear Octree according to the storage structure. The regular Octree needs to store 8 pointers of the sub node and 8 pointers of the parent node, and the non-leaf node needs to store the next sub node pointer, also, the storage efficiency is low [7]. But linear Octree is a efficient storage structure.It uses 8 binary prefix code, that is to say to assign the smallest node value(x, $\mathrm{y}, \mathrm{z}$ ) of the 8 brothers node code is 0 which belong to the same father node, and adjacent sibling node number along the $\mathrm{X}$ direction increased 1, along the $\mathrm{Y}$ direction increased 2 , along the $\mathrm{Z}$ direction increased 4 , also, take the parent of the code as the prefix of the 8 sub node code. In order to ensure the same length of the Octree node code, this paper increases a string special characters from 0 7 octal number after coding. So that it can make each node coding length is the maximum depth of the tree.

\subsection{Cube Subdivision Degree}

It needs a reasonable sub-cube partitioning method to Constructing Octree model. The Cube are finely divided 


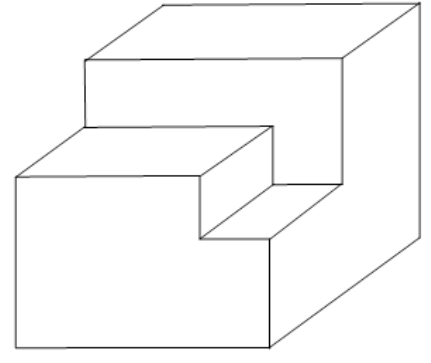

(a) the 3D entity

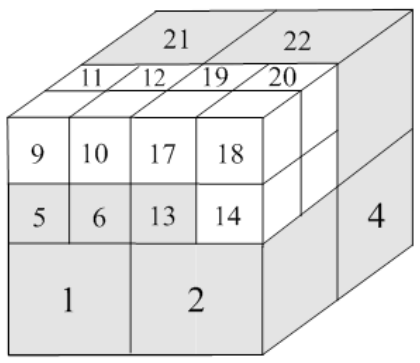

(b) Octree partition

Fig. (1). Octree partition principle.

more, the average data points fall in each cube are less. If the cube partitioning is too fine, so that each data point fall on different cube, there will be a large number of redundant cubes. Octree depth determines the subspace (leaf nodes) point number and the average points which each subspace contains. If set the total number of spatial data is n, and the value of average points is $\mathrm{k}$, so that the leaf node is $\mathrm{n} / \mathrm{k}$, the Octree depth $\mathrm{h}$ can be described as

$\mathrm{h} \geq\left|\log _{8} \frac{\mathrm{n}}{\mathrm{k}}\right|+1$

In the equation (1), the symbol $\|$ means integer operation. Obviously, the depth of the tree is minimum when the Octree is filled. There are two common methods used for cut apart the sub-cube, one is based on the point density to set threshold [8], the other is based on the side length of sub-cube to divide Octree [9]. The $\mathrm{k}$ is an average value of each cube point, and it can reflects the intensive degree of points. It can set the value of $\mathrm{k}$ according to the total data (n) and depth (h) of Octree. It will take $\mathrm{k}$ as the condition threshold of the end of division, to check each sub-cube when subdividing. If the number of sub-cube points is more than $\mathrm{k}$, it will stop dividing. If not, it needs to continue to dividing until every subcube point satisfies the threshold.

The space bounding box of point data is divided into many sub-cubes which length is specified. After divided, there is a number of point data in each sub-cube, and to streamlining data by the iterative calculation in the subcubes. So that, after compressed, the point data density is moderate, besides, it can efficiently obtain information of each point in the local geometry.

One of the basic principles of surface parameterization is to ensure the surface has point value in each interval. That is to say, the parameterization is uniform, and it can avoid the singularity. For some complex surface shape, the above two methods of segmentation don't control well the depth of the Octree. If all the points fall within a bounding box, so that the segmentation of Octree is imbalance, and it is not conducive to searching calculation [10]. In this paper, it is to control points based on the curvature of the shape function, and to improve the traditional Octree generation algorithm, take the shape function $\mathrm{r}(\mathrm{x}, \mathrm{y})$ as a branch threshold function with the node splitting, so that it can streamline the nodes of the Octree. In this way, it can ensure the balance of Octree, and improve the efficiency of the algorithm of time and space.

The sampling density depends on the surface curvature. The larger curvature is, the greater the sampling points should be closer. On the contrary, the smaller the curvature is, the rarer the sampling point is. Set Q as the surface sampling point, the $\mathrm{q}(\mathrm{x}, \mathrm{y}, \mathrm{z})$ is a sampling point for $\mathrm{Q}$, and the $\mathrm{f}$ $(x, y)$ is the interpolation on the surface point of Q. $r(x, y)$ is a shape function to reflect the surface curvature of $f(x, y)$. Obviously, the larger shape function $\mathrm{r}(\mathrm{x}, \mathrm{y})$ is, the more sampling points are. Therefore, the shape function $\mathrm{r}(\mathrm{x}, \mathrm{y})$ can be taken as a condition of whether to continue the sub-cube splitting. Considering the invariance of the grid, the shape function $r(x, y)$ is should only depended on the curvature of the surface inherent measure [11], as follows

$r(x, y)=\frac{k(x, y)-\min _{x, y} k(x, y)}{\max _{x, y} k(x, y)-\min _{x, y} k(x, y)}+c$

Among them, $\mathrm{c}>0$, as constant, $\mathrm{k}(\mathrm{x}, \mathrm{y})$ is the geometric average of the main curvature, as follows

$\mathrm{k}(\mathrm{x}, \mathrm{y})=\frac{\left(\mathrm{f}_{\mathrm{xx}}^{2}+\mathrm{f}_{\mathrm{yy}}^{2}\right)^{1 / 2}}{\left(1+\mathrm{f}_{\mathrm{x}}^{2}+\mathrm{f}_{\mathrm{y}}^{2}\right)^{3 / 2}}$

The basic steps of Octree generation algorithm as follows

Calculating the overall points $\mathrm{n}$ of space, set the average points $\mathrm{k}$ of the subspace leaf node, and estimate the depth $\mathrm{h}$ of the Octree according to the function (1).

Calculate the minimum bounding box which containing all the point, take it as the range of the Octree root node. According to the breadth-first manner of establish Octree, to store the corresponding node information.

Calculate the function $\mathrm{r}(\mathrm{x}, \mathrm{y})$ of sampling point in the cube. If it meets the branch threshold function values, so that stop dividing, but if not, it will divide the sub-cube into $8 \mathrm{sub}$ nodes.

In the process of Octree dividing, take the stop splitting nodes as a leaf, and store the code which belongs to the local surface.

Repeat the step of (4) and (5), until stopping the subdividing of all sub-cubes. In order to control the depth of the 


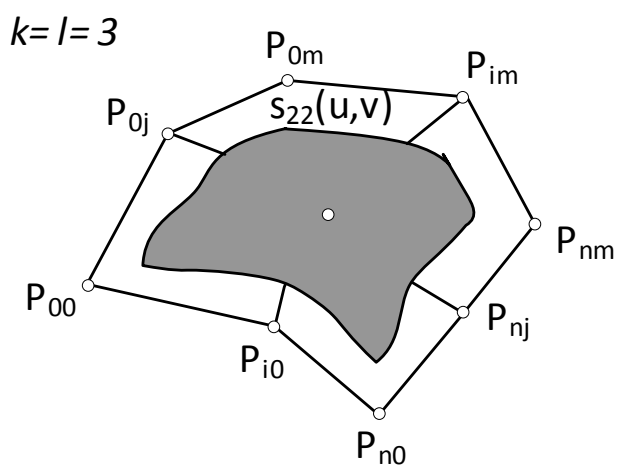

Fig. (2). NURBS surface fitting.

Octree, when it's 2 times deeper than the estimate depth $\mathrm{h}$ (minimum), it also stop dividing.

\section{HYBRID DATA MODEL}

\subsection{NURBS Surface Representation}

The basic idea of the parameter function is to use the limited space data to structure a function, and the new space is generated by the analytic expression in order to close to the original entity. It is difficult to use analytic function to express the complex space objects. In order to overcome the limitations of analytic function, it has put forward a nonanalytic function. The non-uniform rational b-spline (NURBS) function is developed based on B spline function. It is widely used in engineering design, in recent years. It has become a rehashed research in the field of 3D modeling.

As shown in Fig. (2), the rational function of $\mathrm{k}, 1$ NURBS surface is represented as

$$
S(u, v)=\frac{\sum_{i=0}^{n} \sum_{j=0}^{m} w_{i j} P_{i j} N_{i}^{k}(u) N_{j}^{l}(v}{\sum_{i=0}^{n} \sum_{j=0}^{m} w_{i j} N_{i}^{k}(u) N_{j}^{l}(v)}
$$

In the function, $P_{i j}(0 \leq i<n, 0 \leq j<m)$ is the control vertices, and they form a control network. $w_{i j}(0 \leq i<n, 0 \leq j<m)$ is the weight factor of the control point $P_{i j} . N_{i}^{k}(u)(0 \leq i \leq n)$ and $N_{j}^{l}(v)(0 \leq j \leq m)$ respectively are k times, 1 times b-spline basis function of the $\mathrm{u}, \mathrm{v}$ direction. They are respectively nonuniform node vector of $\mathrm{u}$ and $\mathrm{v}$ direction.

$$
\begin{gathered}
U=\left\{u_{0}, \ldots, u_{n+k} \mid u_{i} \leq u_{i+1}, \quad i=0, \ldots, n+k-1\right\} \\
V=\left\{v_{0}, \ldots, v_{m+l} \mid v_{j} \leq v_{j+1}, \quad i=0, \ldots, m+l-1\right\}
\end{gathered}
$$

They are decided by De-Boer recursion formula.

The NURBS method can be used to express surface by the unified mathematical form, and it has the weight factor to influence the surface shape, so that it can be used to make the shape is easy to be controlled and came true.
The NURBS surface fitting has the characteristics of the local support, the smooth continuity, and the space geometrical invariability. But the NURBS method has its obvious shortcomings [12], When it take the method of one by one piece construction to represent complex topology structure, it needs to cut surface, and it wants to consider the smooth stitching between piece and piece. Therefore, it is difficult to keep the joint smooth.

\subsection{Mixed Data Structure}

In the model of the mixed data, the three-dimensional space division can be carried out on the entity by using Octree, and the irregular surface can be matched by using NURBS. If the sub-cube after divided is located in the boundary of the entity and the outline is not a regular cube, the NURBS surface can be used to describe the surface of volume element. The information of surface, edge and vertex of the sub-cubes should be added into the boundary node, in order to form an expanded Octree structure. As is shown in Fig. (3), the circle means the cube is not full filled by a target, the gray rectangle means the cube is full filled by a target, the blank rectangle means there is no target in the cube and the small triangle-gray rectangle means the cube is a boundary of NURBS surface.

The logical structure adopts an expanded OctreeQuadtree mixed structure. The mixed data structure consists of data structure and surface structure. The data structure adopts Octree structure and surface structure adopts Quadtree structure. The surface coding property of the Octree leaf node structure data refers to the related surface of the node. The Quad-tree surface does not store the data of leaf node. All geometric data of the data structure leaf node related to surface also belongs to surface structure. The last structure node of the surface structure Quad-tree is linked to the leaf node of the data structure Octree. In another words, the last node of surface structure needs to store four corresponding pointers of leaf node into the data structure and establishes relation between surface and data points. It controls point data which provided by surface structure according to Octree leaf node, and the data structure can be matched by using NURBS.

C programming language of the mixed data structure describe as follows: 



Fig. (3). Octree-NURBS mixed structure.

struct OctreeNode $\{\quad / /$ data layer Octree

float $\mathrm{x}, \mathrm{y}, \mathrm{z} ; \quad / /$ sampling point data

long code; // node coding

int size; // node size

int type; // property

int layer; // node layer

int index; // No. Of node in brother nodes

float $r / /$ shape function of surface

struct OctreeNode * pParent; //father node pointer

struct OctreeeNode * PChd[8]; //child node point-

er

bool leaf; // leaf child node or not

bool Polygon; // linked to NURBS surface or not

NurbsPoint** point; // NURBS control point int nNurebsCtrl; // NURBS control points num Nurbs* pNurbs; // relevant NURBS surface point-

er

int NurbsObjectID; // ID code of surface object

\}

Struct QuadTree \{\} \{ // surface layer Quad-tree

String code; // node coding: partial surface coding

int layer; // node layer

int index; // No. Of node in brother nodes

struct QuadNode *father; // father node pointer

struct QuadNode *child[4]; // child node pointer

struct OctreeNode *point; //pointer of Octree leaf

\}

\subsection{Surface Reconstruction}

As to a complex-shape surface model, it is difficult to realize overall match with NURBS. The reason is that the scat- tered point data can not establish the rectangular array [13]. The point data is divided into different areas according to the features of entity prototype, and the different surfaces are matched by data points of different areas. Four leaf nodes which based on Octree constitute a NURBS surface quadrilateral area. The other control points are generated according to a fixed characteristic quantity or the topological relation between data points [14]. After dividing point data into a series of grids, and to reconstruct the NURBS surface on the grids.

The surface is established according to point data. Space neighbor division [9] should be carried out for data. Suppose the space neighbor coordinate of the child cube of the data point $q(x, y, z)$ is $(\mathrm{a}, \mathrm{b}, \mathrm{c}), q=q_{n-1} \ldots q_{i} \ldots q_{1} q_{0}$ is the code of Octree node corresponding to child cube. Each code qi is octal numeral. $\mathrm{q}_{0}$ to $\mathrm{q}_{\mathrm{n}-1}$ means the path from leaf node to root. Space neighbor coordinate $(a, b, c)$ of the child cube of the data point can be calculated if the code $q$ of Octree node of child cube has been. As is shown in formula (7).

$$
\begin{aligned}
& \mathrm{a}=\sum_{\mathrm{i}=1}^{\mathrm{n}-1}\left(\mathrm{q}_{\mathrm{i}} \% 2\right) * 2^{\mathrm{i}} \\
& \mathrm{b}=\sum_{\mathrm{i}=1}^{\mathrm{n}-1}\left(\left[\frac{\mathrm{q}_{\mathrm{i}}}{2}\right] \% 2\right) * 2^{\mathrm{i}} \\
& \mathrm{c}=\sum_{\mathrm{i}=1}^{\mathrm{n}-1}\left(\left[\frac{\mathrm{q}_{\mathrm{i}}}{4}\right] \% 2\right) * 2^{\mathrm{i}}
\end{aligned}
$$

The coordinate of Octree leaf node can be calculated according to the formula (7). The leaf nodes are four control points of hook face reconstruction, other control points can 

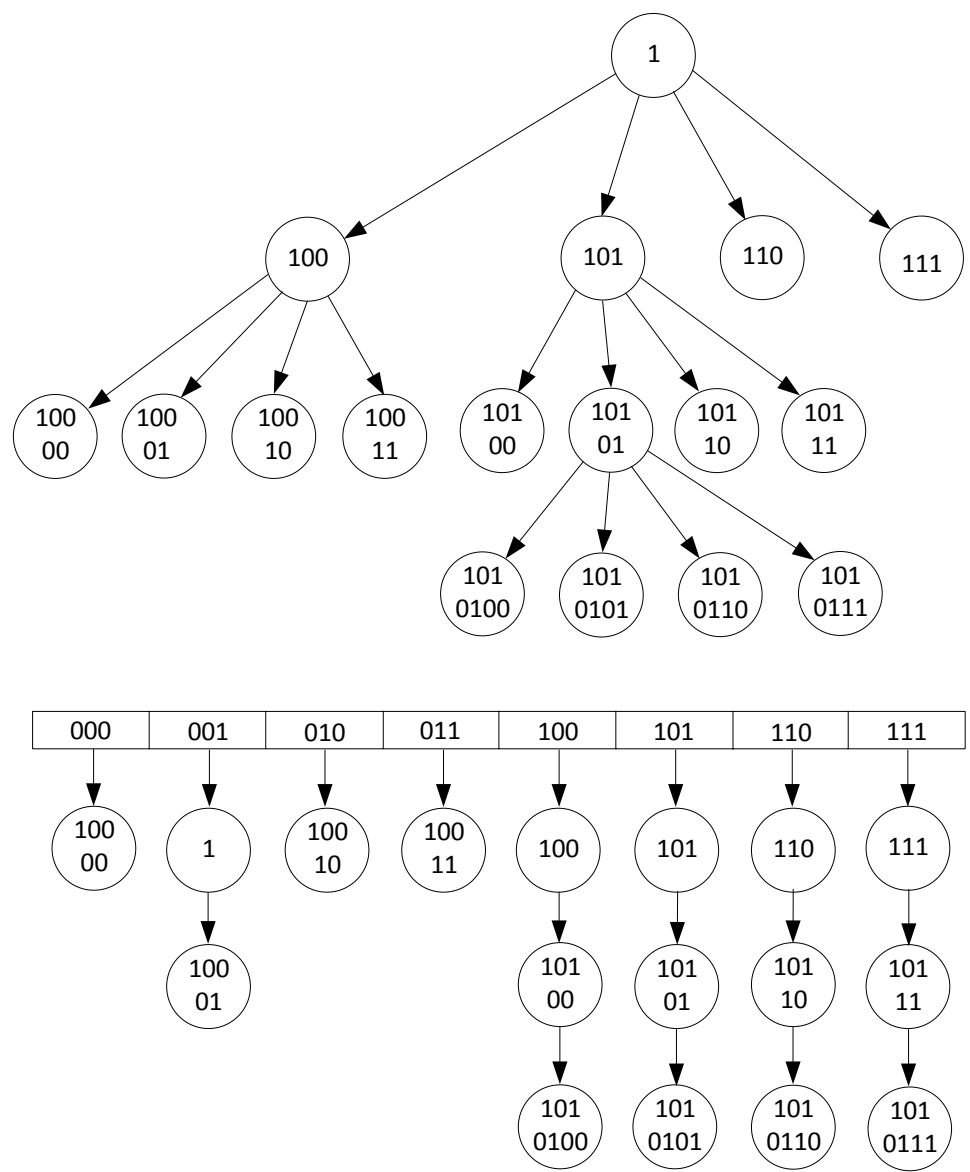

Fig. (4). Structure of hook face Quad-tree.

calculated by using spline basis function. The control point of NURBS hook face is given to be $\mathrm{P}_{\mathrm{ij}}$, and then the control peaks of approximate coplanar of four angles are $\mathrm{P}_{00}, \mathrm{P}_{0 \mathrm{~m}}$, $\mathrm{P}_{\mathrm{n} 0}$ and $\mathrm{P}_{\mathrm{nm}}$. The normal vectors of approximate plane of four angular points are:

$$
\begin{aligned}
& \mathrm{N}=\mathrm{v}_{0} \times \mathrm{v}_{1}+\mathrm{v}_{1} \times \mathrm{v}_{2}+\mathrm{v}_{2} \times \mathrm{v}_{3}+\mathrm{v}_{3} \times \mathrm{v}_{0} \\
& \mathrm{v}_{0}=\mathrm{P}_{0 \mathrm{~m}}-\mathrm{P}_{00} \\
& \mathrm{v}_{1}=\mathrm{P}_{00}-\mathrm{P}_{\mathrm{n} 0} \\
& \mathrm{v}_{2}=\mathrm{P}_{\mathrm{n} 0}-\mathrm{P}_{\mathrm{nm}} \\
& \mathrm{v}_{3}=\mathrm{P}_{\mathrm{nm}}-\mathrm{P}_{0 \mathrm{~m}}
\end{aligned}
$$

Normalization processing:

$$
n=(A, B, C)=\frac{N}{|N|}
$$

$\mathrm{A}^{2}+\mathrm{B}^{2}+\mathrm{C}^{2}=1$

The point of approximate plane is equal to the vector average $\mathrm{P}$ of four angular points. The approximate plane equation is:

$$
\begin{aligned}
& \mathrm{P}=\left(\mathrm{P}_{00}+\mathrm{P}_{0 \mathrm{~m}}+\mathrm{P}_{\mathrm{n} 0}+\mathrm{P}_{\mathrm{nm}}\right) / 4 \\
& \mathrm{D}=-\mathrm{nP} \\
& \mathrm{Ax}+\mathrm{By}+\mathrm{Cz}+\mathrm{D}=0
\end{aligned}
$$

After generating NURBS hook face, four NURBS hook faces form a leaf node of Quad-tree, one hook recursion construction is formed through the pointer. Each four small quadrangle grids corresponding to the control grid has a common point, which are the interaction points of four grids. As to each common interaction points, a new grid is generated after establishing a link with the node of Quad-tree. The old grid can be expressed as a new grid after each merger. The grid can be restrained to a composite hook face after constant mergers.

The construction of the hook face Quad-tree is generated by the way of postorder traversal, in another words, combining daughter hook face gradually by the way of postorder traversal, after getting a hook face expressing method of Quad-tree construction, a mark is set with root pointer. The whole hook face is displayed gradually by the way of postorder traversal Quad-tree while drawing. The mixed model establishes a hook face index through the hook face coding property of data layer Octree. The coder of middle layer node adopts prefix coding [15]. The last three of the coding is set as the key words, and then a Hash table is constructed by using chain address to process conflict. It is shown in Fig. (4).

\section{TEST RESULT AND ANALYSIS}

A model system is developed by using Visual $\mathrm{C}++6.0$. $3 \mathrm{D}$ modeling and visualization of entity can be realized by 


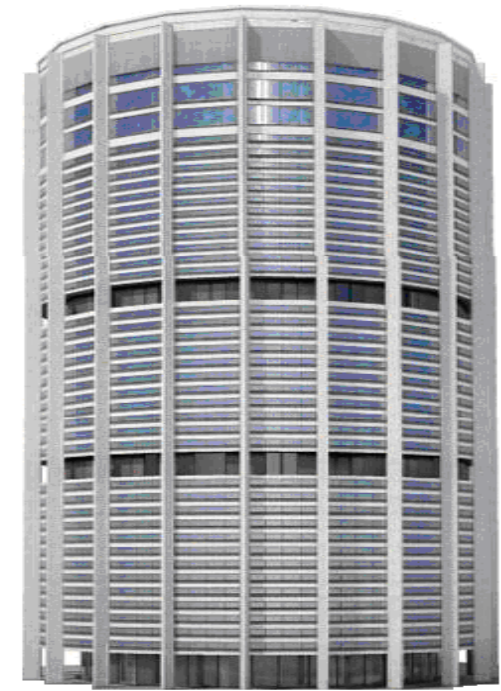

Fig. (5). Test result.

Table 1. Algorithm running time (s).

\begin{tabular}{|c|c|c|c|}
\hline Object & $\begin{array}{c}\text { Data } \\
\text { points No. }\end{array}$ & $\begin{array}{c}\text { Conventional } \\
\text { algorithm }\end{array}$ & Our algorithm \\
\hline \hline Spheroid & 519000 & 182.64 & 4.75 \\
\hline Dragon & 287000 & 29.38 & 4.19 \\
\hline Buddha & 215000 & 9.26 & 3.91 \\
\hline
\end{tabular}

using OpenGL technology. OpenGL supports $\mathrm{C} / \mathrm{C}++$ language programming environment. More than 100 key functions and assistant functions can be provided. It has the strong function on the aspect of 3D modeling and visualization, including light set, Texture mapping, projection transformation, visual conversion, etc. Fig. (5) is the visualization result of a model.

To verify the effectiveness of model and algorithm, computer test has been done. The reconstruction algorithm put forward in the article is compared to the conventional algorithm [16]. Table 1 shows the model reconstruction time of the conventional algorithm and the algorithm put forward in the article with high resolution. From the test result, the time efficiency of the reconstruction algorithm put forward in the article is superior to the conventional algorithm. Especially for the models of some irregular hook faces, the reconstruction efficiency can be improved to be 40 times. In addition, on the premise of the same resolution, the data storing space of the mixed model can be saved $30 \%$. The mixed model has quicker and better reconstruction efficiency. It is suitable for real-time drawing and surface reconstruction of large amount of data models.

\section{CONCLUSION}

3D modeling is applied in many fields so that it needs further research. A mixed data model based on Octree,
Quad-tree and NURBS is put forward on the basis of researching 3D modeling theory and method. The Hash table is established based on Octal prefix encoding by using Octree compact 3D space and irregular hook face of entity described through NURBS. The Octree leaf node linked to be Quadtree leaf node reduces the storing space of model. From the test, the mixed model saves the data storing space and improves the reconstruction efficiency effectively. The model can be used for 3D visualization of the entity. While constructing hook face, the accuracy and efficiency of the model reconstruction algorithm are controlled by the scale of the point data. Theory and algorithm need to be researched further.

\section{CONFLICT OF INTEREST}

The authors confirm that this article content has no conflict of interest.

\section{ACKNOWLEDGEMENTS}

The study was financially supported by National Natural Science Foundation of China (NSFC)(No. 61271369), and Beijing City Finance Special for Specialty Construction such as Computer Science and Technology Specialty (No. PXM2014_014209_07_000009). 


\section{REFERENCES}

[1] M. Yang, "Research on approaches to automatic 3D reconstruction parametric modeling of architectures based on image," $\mathrm{Ph}$. D. thesis, Ocean University of China, Qingdao, CHN, 2009.

[2] R. Guo, C. Liu, and W. F. Ding, "Application of extended Octree Model in the 3D display system," Mini-Micro computer system, vol. 31, pp. 373-376, Feb 2010

[3] Y. B. Jing, L. Wang, and L. Bi, "Segment model modeling algorithm of complex ore body," Journal of Huazhong university of science and technology (JCR Science Edition), vol. 38, pp. 97-100, Feb 2010.

[4] H. C. Ma, and Z. Y. Wang, "Distributed data organization and parallel data retrieval methods for huge laser scanner point clouds," Computers and Geosciences, vol. 37, pp. 193-201, Feb 2011.

[5] H. Fu, L. Liang, and F. Wang, "A point cloud segmentation algorithm using local convexity and Octree," Journal of Xi'an Jiaotong University, vol. 46, pp. 60-65, Oct 2012.

[6] X. M. Gao, and M. Y. Pang, "Incremental mesh reconstruction from unorganized points," Mini-Micro computer system, vol. 32, pp. 2096-2100, Oct 2011.

[7] M. Wang, and Y. H. Tseng, "Incremental segmentation of lidar point clouds with an Octree-structured voxel space," The Photogrammetric Record, vol. 26, pp. 32-57, 2011.

[8] J. Wu, J. Yang, and H. X. Qin, "Incremental surface reconstruction of unorganized points based on BFS," Journal of Shanghai Jiaotong University, vol. 42, pp. 1740-1744, Oct 2008.
[9] Z. W. Shao, and P. Xi, "Data reduction for point cloud using Octree coding," Journal of Engineering Graphics, vol. 31, pp. 73-76, Apr 2010.

[10] A. Jagannathan, and E. L. Miller, "Three-dimensional surface mesh segmentation using curvedness-based region growing approach," IEEE Transactions on Pattern Analysis and Machine Intelligence, vol. 29, pp. 2195-2204, Dec 2007.

[11] X. M. Wang, and J. X. Liu, "A new approach of adaptive compression and mesh generation for large scale scattered data," Journal of Engineering Graphics, vol. 31, pp. 92-95, Feb 2010.

[12] S. Y. Chen, and Q. Guan, "Parametric shape representation by a deformable nurbs model for cardiac functional measurements," IEEE Transactions on Magnetics, vol. 58, pp. 480-487, Mar 2011.

[13] Y. Bai, X. Han, and J. L. Prince, "Digital topology on adaptive octree grids," Journal of Mathematical Imaging and Vision, vol. 34, pp. 165-184, 2009.

[14] J. Nie, Z. Ma, and Y. Hu, "Rapid surface reconstruction algorithm from dense point cloud," Journal of Computer Aided Design and Graphics, vol. 24, pp. 574-582, May 2012.

[15] T. Lewiner, V. Mello, and A. Peixoto, "Fast generation of pointerless octree duals," Journal Compilation, vol. 29, pp. 1661-1669, May 2010

[16] Q. Du, and D. S. Wang, "Constrained boundary recovery for three dimensional delaunay triangulations," International Journal for Numerical Methods in Engineering, vol. 61, pp. 1471-1500, Sep 2004.

(C) Yujian et al.; Licensee Bentham Open.

This is an open access article licensed under the terms of the Creative Commons Attribution Non-Commercial License (http://creativecommons.org/licenses/by$\mathrm{nc} / 3.0 /$ ) which permits unrestricted, non-commercial use, distribution and reproduction in any medium, provided the work is properly cited. 